\title{
André Beauchamp
}

Consultant en environnement -

Membre de la Chaire de recherche en éthique de l'environnement

- Hydro-Québec/McGill

(1979)

\section{"Séparation et culture: quelques retentissements éthiques"}

Un document produit en version numérique par Jean-Marie Tremblay, bénévole, professeur de sociologie au Cégep de Chicoutimi

Courriel: jmt_sociologue@videotron.ca

Site web pédagogique : http://www.uqac.ca/jmt-sociologue/

Dans le cadre de la collection: "Les classiques des sciences sociales"

Site web: $\underline{\text { http://www.uqac.ca/Classiques des_sciences_sociales }}$

Une collection développée en collaboration avec la Bibliothèque

Paul-Émile-Boulet de l'Université du Québec à Chicoutimi

Site web: http://bibliotheque.uqac.uquebec.ca/index.htm 
Cette édition électronique a été réalisée par Jean-Marie Tremblay, bénévole, professeur de sociologie au Cégep de Chicoutimi à partir de :

André BEAUCHAMP, "Séparation et culture : quelques retentissements éthiques". Un article publié dans l'ouvrage collectif, Le référendum: un enjeu collectif, pp. 109-130. Montréal: Éditions Fides, 1979, 174 pp. Collection: Cahiers de recherche éthique, $n^{\circ} 7$.

M. Beauchamp est consultant en environnement - Membre de la Chaire de recherche en éthique de l'environnement - Hydro-Québec/McGill.

[Autorisation formelle accordée par la directrice adjointe de la revue L'Action Nationale le 9 mars 2004.]

S1

Courriels : revue@action-nationale.qc.ca ou abeauchamp@cjf.qc.ca

Polices de caractères utilisée :

Pour le texte: Times, 12 points.

Pour les citations : Times 10 points.

Pour les notes de bas de page : Times, 10 points.

Édition électronique réalisée avec le traitement de textes Microsoft Word 2001 pour Macintosh.

Mise en page sur papier format

LETTRE (US letter), 8.5' x 11'”)

Édition numérique réalisée le 22 octobre 2004 à Chicoutimi, Ville de Saguenay, province de Québec, Canada. 


\title{
Table des matières
}

\author{
Introduction
}

1. Le point de vue canadien

2. Le point de vue francophone québécois

3. Le discours éthique

4. Discours politique, discours éthique ?

5. Discours politiques et arguments moraux

a) Arguments fédéralistes

6. La question de fond

7. Des développements ultérieurs ? 


\section{Introduction}

André Beauchamp est prêtre du diocèse de Montréal. Il a travaillé depuis dix ans dans le domaine de l'éducation de la foi des adultes, en se consacrant plus spécialement au rapport de la foi avec les questions socio-politiques. Engagé activement dans les milieux nationalistes, il a été président de la Société SaintJean-Baptiste de Montréal et en est maintenant vice-président. Il est actuellement responsable du module Éducation en environnement, au Service de protection de l'environnement.

$\underline{\text { Retour à la table des matières }}$

Il est bien rare qu'on ait à choisir un pays. « Les États-Unis sont nés d'une révolution. Beaucoup de pays européens aussi. En Afrique, il y a tout un monde entre les pays qui ont acquis leur indépendance en luttant et les autres, Au Canada, notre histoire est à l'inverse de cette expérience. Les loyalistes anglais ont refusé la révolution américaine. Notre chrétienté québécoise a rejeté la révolution française. Beaucoup d'allophones ont quitté leur pays à cause d'une révolution » (Jacques Grand'Maison, Le Devoir, 6 juillet 1978).

\section{Le point de vue canadien}

$\underline{\text { Retour à la table des matières }}$

En un sens, la question que pose le séparatisme québécois est scandaleuse et, pour quelqu'un qui ne connaît pas bien l'histoire d'un point de vue de francophone, elle est inimaginable. Car indubitablement le Canada est un pays où l'on se porte bien : bon niveau de vie, croissance économique, histoire paisible, tensions sociales modérées. On peut comprendre la phrase de Pierre Elliott Trudeau au lendemain de l'élection du P.Q. : «Diviser le Canada c'est 
un péché contre l'humanité, un péché contre l'Esprit. » Par exemple, le Canada est un pays sans guerre, sauf deux épisodes avec les États-Unis en $1775 \mathrm{au}$ moment de la création des États-Unis, et en 1812. Mais dans les deux cas, le Canada n'existait pas encore dans sa forme actuelle. Pour le reste, trois mille milles de frontière commune avec les États-Unis sans le moindre accrochage. Même chose au Nord avec l'U.R.S.S. Les deux occasions où le Canada a été impliqué furent les deux grandes guerres mondiales par le biais de son lien privilégié à l'Angleterre. Mais jamais le sol canadien n'a été vraiment menacé. Il s'ensuit presque que le Canada est un pays sans histoire, un pays fait à l'amiable sans qu'une grande passion ne l'ait porté, sans qu'une dramatique ne l'ait traversé.

Le Canada pourrait être un modèle de pays pacifique, ou mieux un exemple illustrant qu'un pays puisse n'être qu'un système d'administration et de cohérence sans devenir une patrie. L'analogie à la famille pourrait être éclairante. Il est des familles unies et passionnées de leur identité. Il en est d'autres qui se contentent d'être un dortoir, un lieu commode de référence. À première vue, le Canada serait de ce type.

Au plan du discours, mettre le Canada en question est donc un acte banal puisque le Canada ne serait qu'un pacte administratif révisible, à juger au mérite. Par ailleurs, si la réussite est si brillante, le mettre en question n'est-ce pas faire oeuvre d'imprudence?

\section{Le point de vue francophone québécois}

$\underline{\text { Retour à la table des matières }}$

Voilà donc une façon de voir. Mais déplaçons la question. Prenons-la, par exemple, du point de vue des Indiens. On ne peut pas en parler comme d'un peuple unifié qui aurait bâti un pays et édifié une culture. Mais il y avait des groupes humains cohérents occupant des territoires et définissant des cultures autonomes, avec chacune leur langue ou dialecte, leur histoire, leur musique, leurs mythes, leurs façons de vivre et de donner sens à l'existence. D'un point de vue autochtone, l'histoire du Canada est l'histoire d'un colonialisme violent puisque le territoire a été occupé de force par d'autres et les Populations indiennes progressivement éliminées et réduites à des « réserves». On peut bien argumenter et dire par exemple que le colonisateur francophone a établi de meilleures relations avec l'Indien (meilleurs contacts, exploitation économique modérée, échanges culturels, métissage) mais c'est une affirmation 
délicate et sujette à caution. L'homme blanc est un violent qui a spolié l'Indien de son sol et de son âme. Et les traités, les ententes, les droits reconnus aux Indiens ne sont pas nécessairement la promesse d'un avenir pacifique. Par rapport à cette réalité, le discours canadien de l'unité est une masque idéologique qui camoufle une praxis dont la justice est plus que douteuse.

D'un point de vue de francophone québécois, quel est donc le sentiment de l'appartenance canadienne ? Voyons d'abord la dynamique des mots. Les habitants d'ici fondèrent la Nouvelle-France. Puis, quand la conscience de l'habitant canadien se fut affirmée en opposition à la métropole parisienne, surgit le nom de Canada, distinct de la France et des colonies anglaises. Le Canadien, à l'origine, c'est le francophone. Pour le Canadien, le traumatisme historique c'est la conquête anglaise. Le Canadien avait conscience de l'empire de l'Amérique française, de la Louisiane aux Prairies, avec comme berceau Québec. Alors que le Canadien était venu pour s'implanter et construire un pays, il est conquis par des forces militaires qui sont, à ses yeux, des forces impérialistes et qui résident ici sans y habiter, le temps d'une administration, d'une occupation, d'un service militaire. On ne parle pas alors de Canadiens français et anglais, mais de Canadiens et d'Anglais, au sens d'Anglais d'Angleterre. Perception que confirme l'arrivée massive des loyalistes anglais indignés de la révolution américaine.

C'est à mesure que s'impose le concept du Canada (Haut et Bas-Canada, Acte d'Union, Confédération) que les anglophones s'attribuent le nom de «Canadians », nous obligeant à distinguer Canadiens français et Canadiens anglais. S'il y a eu une chance historique canadienne dans l'hypothèse des deux peuples fondateurs et des deux langues, elle a existé de 1867 jusqu'au début du siècle quand les francophones, dont l'appartenance était essentiellement québécoise, ont émigré en masse. Quand ils émigraient aux U.S.A., ils acceptaient de s'assimiler à plus ou moins brève échéance. C'étaient les règles du jeu. En émigrant ailleurs au Canada, en ouvrant l'Ouest, ils croyaient à la réalité canadienne francophone. La question des écoles françaises en Alberta et au Manitoba, la question des Métis, la constitution du visage juridique, politique et économique de l'Ontario et de l'Ouest ne laissaient aucun doute : il n'y a pas de Canada bilingue, bi-culturel, bi-national. Il y a un Canada anglophone et un malaise canadien-français.

La montée du nationalisme francophone québécois est à la fois la reprise du nationalisme canadien-français et sa rupture. Reprise, car il y a filiation évidente. Rupture, car l'analyse sociale et politique amène à conclure qu'il ne suffit plus de militer sur une base canadienne pour obtenir l'égalité et les conditions essentielles à l'épanouissement. Pour un indépendantiste, la base canadienne joue inévitablement (par le jeu politique, économique, culturel) dans le sens de la minorisation des francophones, de leur assimilation, de leur sujétion sociale et politique. Tant d'enquêtes l'ont montré. Seul le Québec peut servir d'horizon à la construction d'un avenir pour les francophones à partir d'un instrument collectif fort et de la récupération de l'ensemble des pouvoirs 
juridiques et politiques. Cela s'appelle un Québec indépendant qui oblige à redéfinir l'unité canadienne.

\section{Le discours éthique}

$\underline{\text { Retour à la table des matières }}$

C'est sur ce fond d'interprétation historique et politique que veut jouer le discours éthique. Je ne suis pas de la thèse de ceux qui prétendent que la morale se perd, que les valeurs auxquelles les gens étaient attachés ont été balayées radicalement et que nous voici maintenant devant un immense vide éthique. On s'empresse alors de citer l'érosion des conduites privées, notamment dans le domaine privilégié des conduites sexuelles. Ou bien l'on déplore la chute de l'autorité, l'effritement de la responsabilité personnelle, la montée vertigineuse du vol, de la fraude, du mensonge, du parjure, l'individualisme moderne ou, le pire de tous, le glissement dans la société de consommation qui rend l'homme dissemblable à lui-même et le fait certainement mourir.

Chacun de nous connaît le ton de ce diagnostic pessimiste.

Mon impression à moi, c'est que l'aspiration morale actuelle n'est pas moindre que celle d'hier. Mais nous sommes en recherche de références. Nous assistons à un déplacement de valeurs et tout le système des normes, si lentement et péniblement élaboré, s'en trouve en porte-à-faux. Ce serait bien vite dit que de traiter de médiocres ou de pervers les gens d'aujourd'hui et de forts, courageux et honnêtes les gens d'hier. Mais les gens d'hier savaient par cœur les conduites qui déterminaient la bonne vie morale alors qu'un scepticisme radical nous a envahis. Sans doute, la médiocrité et la paresse latentes en chacun de nous prennent-elles prétexte du conflit des normes pour en profiter un brin. Mais un recours aussi simpliste ne suffit pas à tout expliquer. Car l'homme est un animal éthique. Il a besoin d'articuler sa responsabilité sur la liberté et sur des motivations qui réfèrent à des critères, des principes, des valeurs. Les premières études sociologiques dans les sociétés scandinaves avaient montré qu'en période d'anomie le taux de suicide avait tendance à grimper à vive allure, comme si les gens, faute de références et de codes, en arrivaient à se punir eux-mêmes pour leur conduite répréhensible ou inqualifiable.

Si l'être humain génère ainsi une anxiété aussi profonde quand il ne sait plus à quoi se référer ni comment orienter ses actions, on doit conclure que l'aspiration morale est, chez lui, absolument fondamentale. En réalité, nous avons moins besoin d'être sermonnés pour nos démissions et nos infractions à 
l'égard des codes d'hier désuets et désormais si vertement dénoncés, si peu cohérents avec nos expériences actuelles qu'on les sent vétustes et insignifiants, qu'encouragés à définir pour demain une morale ouverte et flexible qui convienne aux appels de la situation.

\section{Discours politique, discours éthique?}

$\underline{\text { Retour à la table des matières }}$

Selon moi, l'anxiété morale n'a pas diminué, loin de là. Et les nouveaux moralistes sont légion, pas moins moralisateurs que ceux d'autrefois. Seulement les ténors ont changé ainsi que la salle de concert. Ce ne sont plus les philosophes, les théologiens, les chefs religieux qui tiennent le discours moral. Ce sont plutôt des poètes, des politiciens, des groupes de pression, des publicistes. Et les media ne sont plus le traité d'éthique, ni les sermons, mais les harangues publiques, le cinéma, la radio, la télévision. Combien de séries télévisées sont, en fait, des catéchèses sur la manière de se conduire envers sa fille, la façon d'accueillir un homosexuel, d'assumer son divorce, de sentir l'horreur du vol, de la violence, de la trahison? Tout le monde nous fait la morale. Les chansons : " fais ta vie comme ça te dit, c'est tout ce qui compte au fond». La publicité : "l'assurance-vie, c'est le geste le moins égoïste qui soit ». L'État : « va jouer dehors »; « la vie, on s'attache à elle ».

En empruntant une terminologie marxiste, on pourrait affirmer que si la religion est l'opium du peuple, la publicité a bien pris sa relève. Quand nous regardons ce beau groupe de gars et de filles, jeunes et vivants, s'avancer vers nous dans la force de leur âge avec leur puissance de séduction et nous dire : «lève ton verre avec nous, de plus en plus c'est Laurentide » ou bien nous proclamer les gloires de la «50», c'est en réalité une véritable image du Royaume qui se dresse à nos yeux. Ce n'est pas la marche naïvement triomphante des armées de 1914 quand les filles jetaient des fleurs aux soldats qui allaient mourir au front. Il y avait là une dramatique tragique. Dans les réclames évoquées, la bière est la récompense d'un effort, en général lié au travail («ça vaut la peine de farfouiller parce que ça fait des choses à raconter », au sport, à l'épreuve physique. La femme-objet est ensuite donnée en récompense dans une projection de plénitude et d'apaisement qui est celle du Royaume. «Il n'y aura ni pleurs, ni grincements de dents ». Nous sommes en pleine eschatologie. 
Dans le même ordre d'idée, un message publicitaire de MacDonald nous fait découvrir le secret de MacDonald. Le sceau : approuvé Canada (allusion à l'enquête de la Ceco sur la viande avariée «approuvé Québec »), puis la mention «bœuf pur à $100 \%$ ». « De mieux en mieux ». Et la réclame de conclure : « heureux les propriétaires de dents qui croqueront dans...» On croirait entendre la parole d'Évangile : "Heureuses les entrailles qui t'ont porté et le sein qui t'a allaité ». Sauf que dans l'Évangile, l'appel est à la conversion et la vie de la foi. Ici, il se dégrade en sollicitation commerciale.

Il serait d'ailleurs intéressant de poursuivre l'exploitation des thèmes politiques sous-jacents à la publicité. Quand l'image québécoise est bonne, on la récupère. «La banque d'ici pour les gens d'ici ». Household nous parle de confiance en l'homme à propos d'un prêt consenti à un pêcheur de homards de Grosse-Île. On chuchotte en coulisse qu'il y aurait un pacte des compagnies canadiennes pour projeter à profusion des images de la réalité canadienne. C'est assez évident dans les réclames du $\mathrm{CN}-\mathrm{CP}$ ou dans celles des compagnies de pétrole.

Curieux paradoxe. La publicité se justifie par l'information qu'elle apporte au client et par le dynamisme qu'elle communique à l'économie. Or, en vérité, la publicité c'est plus simplement l'argent au service du mensonge. La part efficace de la publicité, c'est sa charge émotive, le plus souvent inconsciente chez l'éventuel client. C'est un modèle de manipulation. Quand on réussit à vous dire que le Coke est « le vrai de vrai » et que les gens, ayant maintenant ici le goût du vrai et de l'authentique, ne peuvent se retrouver que dans le Coca-Cola, c'est assez pénible.

Or cette même publicité a tendance à devenir le premier véhicule de nos valeurs. "Quand j'étais petit dans mon jardin, je me balançais sur un Michelin qui avait roulé des milliers de milles - pour un Michelin c'est bien facile. Puis quand j'ai eu mon auto-sport, toutes les fins de semaines je montais dans le Nord et changeais de blonde à chaque année - les Michelins eux ont tant duré - Michelin, Michelin, je te confie ma voiture, moi je sais que les autos passent, les Michelins durent -Maintenant que je suis un peu plus usé, marié deux fois puis séparé, je me dis qu'y a pas grand-chose qui dure, à part les pneus Michelin, bien sûr - Michelin... »

Voici un glissement type, et de caractère aliénant, de la notion de fidélité, valeur si difficile de nos jours, à celle de durabilité. Triste société où la fidélité est représentée par les choses et l'infidélité par les personnes. Admettons toutefois que le romantisme faisait cela, en appelant de l'immutabilité du monde à la vanité des sentiments ; mais il avait la décence de ne pas ravaler sa symbolique au niveau de la production mercantile des biens de consommation.

Bonne ou mauvaise, et plutôt les deux à la fois, la publicité semble être devenue le véhicule de notre discours. À l'origine, la publicité s'est modelée sur le discours savant et l'information. Maintenant, le discours savant et 
l'information ont tendance à se modeler sur la publicité. La publicité est une sur-réalité. Comme la pornographie est la substitution de l'image projetée à l'objet sexuel lui-même et à la relation personnelle, de même la publicité devient le substitut symbolique de la réalité, reléguant la réalité elle-même à l'insignifiance.

Situation très ambiguë.

Dans la guerre du référendum, le discours politique devient essentiellement un discours moral. Il en appelle à la rationalité, au bon jugement, à l'évidence. Il se rattache par mille liens aux principes les plus chers de l'humanisme, à la dignité, à la bonté, au souci de vérité et d'authenticité de chacun. Aristote, je crois, disait que la politique est la plus haute activité de l'homme. C'est toujours vrai.

Sauf que le médium mange le message. Pour se dire, le message politique devient publicitaire. Sur un plan éthique, c'est assez éprouvant. «J'ai un beau pays», chante la réclame pour visiter le Canada. Le gouvernement du Québec n'est pas en reste. "Tout le monde s'attache au Québec », dans le jeu de mots de la ceinture de sécurité et de l'amour pour le Québec. «Le Québec est au monde », proclamait le slogan de la fête nationale. Encore un jeu de mots pour signifier la démocratisation de la fête et l'accession du Québec au statut d'individualité politique.

Le 24 juin, le 1er juillet, le 3 juillet, nous avons assisté à la surenchère des fêtes, des spectacles, des artistes «engagés » et des éclosions de joie populaire spontanée très savamment organisées et structurées. Et dire que les membres de la Commission Pépin cherchent une voie sereine et loyale !

On explique cela en affirmant que nous sommes en phase référendaire. Mais le résultat est assez affligeant. Car, ou nous sommes une bande d'imbéciles, ou bien la lutte des clans est si avancée qu'il n'y a plus moyen de faire entendre raison. Dans ce brouhaha, il se peut fort que le discours éthique tenu par et dans le discours politique de l'heure ne soit en réalité qu'un écran de fumée, un alibi. Il s'agirait moins de chercher le vrai que de trouver tous les arguments, y compris celui de la conscience, pour convaincre la marge des indécis.

Si cette hypothèse était correcte, elle dévoilerait le plus formidable assaut fait à la conscience des gens en exploitant leur complexe de culpabilité. Car quoi qu'en dise Marcel Rioux, le Québécois n'a pas laissé sa religion comme on oublie ses mitaines au printemps. Il est tissé de religion et d'impératifs moraux. Mais comme la pratique quotidienne mesurable, vérifiable, s'est dissipée, il ne reste qu'une aspiration confuse et refoulée dans l'inconscient où la propagande politique risque de le renvoyer sans le libérer. Les théologiens disent que le propre de l'espérance, c'est d'inspirer une réforme du réel dans le quotidien. C'est cette quotidienneté qui semble faire défaut maintenant, au 
profit d'un bond prodigieux dans un futur incertain sans que l'anxiété trouve dans l'aujourd'hui les voies de sa guérison.

\section{Discours politiques et arguments moraux}

Acceptons de traiter le discours politique dans son contenu même, dans la valeur de ses arguments sans l'interpréter à un niveau second. Supposons que les prophètes politiques cherchent la vérité de la conscience et non sa manipulation et que leur morale ne soit pas une justification a posteriori de leur choix mais qu'elle précède le mouvement de la conscience pour l'éclairer. Voyons les arguments moraux mis de l'avant dans chacun des deux camps.

\section{a) Arguments fédéralistes}

Retour à la table des matières

Pour un fédéraliste, la séparation du Québec est un acte d'immaturité qui ressemble à une rupture infantile ou adolescente. Si la Confédération canadienne a vécu pendant plus de 100 ans dans l'harmonie des peuples fondateurs, si les francophones ont ainsi survécu et sauvegardé leur langue, certaines de leurs institutions, des droits fondamentaux, c'est qu'ils ont établi une relation dialectique d'opposition et de collaboration, de dialogue et de revendication qui se situe nettement au niveau adulte. Se séparer devient alors un acte de régression psychologique, une fuite du réel, un manque de courage.

Dans la même foulée, il y a l'argument de la charité. On extensionne le concept de la charité fraternelle (amour d'autrui, dialogue, collaboration, pardon des offenses) pour l'appliquer aux communautés ethniques. Certes la communauté francophone a-t-elle beaucoup souffert, et davantage que l'anglophone, du pacte canadien. Mais elle doit apprendre le pardon des offenses et relancer le dialogue plus loin, avec les éléments les plus avancés de la majorité anglophone, avec tous ceux qui sont de bonne volonté.

Cet argument de la charité se déploie en deux volets. D'un côté, si le Québec se sépare, il menace tout le pays car il brise le Canada. Il impose donc sa solution au reste du pays. Il casse le pays en deux, et le Canada anglophone, surtout dans l'ouest, est pris tout entier avec la question de sa propre identité 
face à sa culture canadienne-anglaise tellement influencée par son immense voisin américain. Au cours d'un récent voyage en Alberta, j'ai senti ce malaise qui s'exprimait par ces deux réactions : si vous partez, qu'allons-nous devenir ? si vous partez, nous enverrons l'armée vous mettre au pas.

L'autre type d'argumentation se construit autour de l'idée suivante: qu'adviendra-t-il alors des communautés francophones hors Québec ? N'est-ce pas les rejeter à leurs seules forces et les vouer à l'assimilation? Le Québec peut-il alors se séparer et perdre mémoire des générations de ses descendants qui, en Ontario et au Manitoba, ont transplanté l'arbre français ? Peut-il oublier la branche parallèle d'Acadiens dont le combat au NouveauBrunswick, entre autres, a été beaucoup plus difficile encore que la lutte québécoise?

Dans cette foulée de la charité, on emploie aussi l'argument universaliste. Le monde est aux grands ensembles, aux ententes internationales, au dépassement des ethnies et des petites clôtures que l'histoire a dressées malencontreusement et que la raison doit surmonter. Si l'on veut que l'homme soit un et que l'amour règne, il faut donc penser des entités politiques vastes et complexes dans lesquelles l'individualisme des personnes et des communautés soit enfin dépassé. À l'heure de l'Europe des neuf, du Marché commun, des ententes internationales, de la planétarisation du monde, des vols interplanétaires, il faut parler de l'humanité et non des nations. En ce sens, le Canada est une forme historique préfigurative, pour user du mot de Margaret Mead, tournée vers l'avenir, prospective, alors que le séparatisme serait comme le reliquat historique de la mentalité héritée du 19e siècle et du principe des nationalités qui a inspiré tant de guerres futiles et tant d'oppressions pour des « justes causes ».

Enfin, le dernier argument réfère à la vertu de prudence. Bien ou mal, le Canada a marché pendant 110 ans. Or, l'abandonner au profit d'un hypothétique avenir, n'est-ce pas s'exposer à des désillusions ? Si cela ne marchait pas? Un tien vaut mieux que deux tu l'auras. Ici, quand le discours se détériore en propagande, il glisse vite sur les affres des pensions de vieillesse suspendues et la menace de la crise économique.

Une subtilité de cet argument conservateur consiste à lier le statu quo politique au statu quo des valeurs et de l'idéologie. L'argument devient le danger de l'avenir par rapport aux valeurs du passé. Devant les menaces d'un avenir incertain et si menaçant pour les valeurs établies, la séparation du Québec apparaît comme un saut résolu dans un autre monde et comme la perte de l'héritage des valeurs reçues. Dans une rencontre à Rimouski, j'ai pu me rendre compte qu'un groupe de personnes d'un certain âge voyait la question fédéralisme-séparatisme comme un conflit de génération, Les jeunes sont trop libres sexuellement, sont paresseux, irresponsables, indifférents à leurs devoirs religieux. Cela, c'est la faute du séparatisme et des chefs syndicaux avec lesquels le P.Q. fraie de trop près. C'est toute la même clique de révolutionnaires. En réaction, c'est donc le conservatisme qui va sauver nos valeurs 
et le fédéralisme qui sera le garant de notre héritage. D'une façon plus subtile, on pourrait presque dire : l'armée canadienne va nous aider à mettre nos enfants au pas. Nous avons peur de ce que nos enfants sont devenus. Le voisin va nous donner un coup de main.

\section{b) Arguments séparatistes}

Retour à la table des matières

La panoplie des arguments séparatistes se développe exactement en sens inverse,

Prenant appui sur l'accession à l'indépendance de soixante-dix nations depuis 1945, elle présente la montée du Québec comme une marche en continuité historique avec son passé. Au fond, la vraie lutte qu'ont menée les anciens, ceux de 1791, ceux de 1837, ceux du Bloc populaire ou de l'Union nationale, fut d'assurer au Québec les conditions de sa survie. Tant que le Québec restait de caractère rural, traditionnel, à un stage pré-industriel, sa survivance pouvait s'assurer par un combat local à ras de sol dans chaque communauté. Mais, nous sommes passés d'un nationalisme de survivance à un nationalisme de rattrapage sous Lesage et maintenant à un nationalisme de lutte et d'affirmation.

Donc, loin d'être une régression de l'âge adulte vers une affirmation de soi de type adolescent, la séparation est une maturation et le passage de l'enfance à l'âge adulte. Les comparaisons affluent, de type familial. L'accession du Québec à la souveraineté, c'est comme un fils qui doit se poser en égal à son père pour pouvoir discuter avec lui en vérité. Tant que le Québec se considère comme un nain face à un géant, comme une province face à neuf autres dans une structure fédérale dont la logique est anglo-saxonne, il est en état d'infériorité et il ne peut se situer dans une véritable relation d'autonomie. Pour reprendre le slogan célèbre de Daniel Johnson : égalité ou indépendance. L'indépendance, au moins à titre logique, est la condition de l'égalité ; la relation du père et du fils ne se dénoue que dans la révolte du fils qui se pose comme étranger à son père pour pouvoir ensuite établir avec lui une relation d'adulte profitable aux deux. Dans l'unité canadienne, la minorité francophone n'a jamais été traitée comme une égale. Seule sa subversion (symbolique) peut permettre de construire un vrai pays.

L'indépendance n'est donc pas un caprice. C'est un acte de maturité psychologique. En posant ainsi la question de son identité, le Québec ne brise donc pas la charité, il ne déchire pas la robe sans couture de l'unité canadienne. Il donne au Canada la chance de se définir. Certes, il renvoie au reste du pays l'anxiété de sa propre définition. La question n'est plus «What does Québec want? » mais « What is Canada and why Canada? »e départ des 
fils repose aux parents la question de leur avenir et de leur identité de couple. La brisure du Canada pourrait amorcer une nouvelle définition de l'union canadienne, avec une redéfinition des provinces dans une union ouest-est (I'Île-du-Prince-Édouard peut-elle être encore une province ?) ou de nouveaux alignements nord-sud, la Colombie britannique se rattachant plus naturellement aux U.S.A. par exemple.

La « charité politique » oblige-t-elle à tenir compte du malaise d'identité du Canada sans portion québécoise ? La thèse indépendantiste soutient que non.

Que dire maintenant des obligations du Québec à l'égard des francophones hors Québec ? Les rameaux de la famille francophone ne peuvent pas être plus mal protégés qu'ils ne le sont déjà, au tiers ou à demi assimilés et forcés de travailler dans une autre langue que la leur, confinés à un rôle de réserve culturelle où le français n'est plus l'élément structurant de la culture et de l'identité. La sécession du Québec en fera-t-elle les victimes du ressentiment canadien ? À première vue, on le croirait. Et ce serait donc charité de rester à leur côté dans le combat canadien. Pourtant les tenants séparatistes prétendent que non. Un nouveau radicalisme chez les francophones hors Québec laisse au contraire entrevoir que l'hypothèse séparatiste du Québec renforcit les positions des francophones hors Québec. Pour les séparatistes, charité bien ordonnée commence par soi-même, d'autant plus que la montée du nationalisme québécois raffermit plus qu'elle ne décourage la lutte des autres francophones.

Quant à l'argument de la prudence, les tenants de l'indépendance inversent l'argumentation. Les études, surtout l'enquête Laurendeau-Dunton, montrent que partout au Canada et au Québec les francophones se classent les derniers, si l'on fait exception des Indiens, et que Montréal est la seule grande ville où un unilingue anglophone se tire mieux d'affaires qu'un bilingue. Ce qui est imprudent c'est donc l'unité canadienne puisque, à long terme, elle joue contre les intérêts francophones. La prudence étant la vertu du jugement concret dans une situation donnée, seule l'audace d'une option politique claire peut nous sauver d'une sujétion encore plus grande.

Enfin, la grande thèse morale de l'indépendance, ce serait sa référence prophétique à l'idéologie de la libération. On sait qu'en Amérique du Sud notamment, une idéologie nouvelle est née, issue d'une praxis, celle de la libération. Il s'agit de considérer l'état de fait non pas comme une fatalité, mais comme le résultat de facteurs historiques ayant leur poids et leur signification. La pauvreté en Amérique du Sud n'est qu'une triste réalité. Il s'agit là d'une pauvreté créée par d'autres, entretenue et développée au profit d'économies étrangères ou de minorités possédantes. Le sous-développement est en réalité un sous-développement entretenu. Dans cette situation, il n'y a qu'une praxis, c'est celle de la libération. C'est d'abord une praxis collective, c'est-à-dire celle qui récuse l'arrivisme individuel (je m'en tirerai tout seul, je fais mes salaires, 
je réussis ma vie, même si la condition globale de mon peuple n'a pas changé) et qui met de l'avant des images collectives.

Dans cette hypothèse, il ne s'agit pas de savoir si monsieur ou madame untel ont fait fortune, ont réussi et sont considérés par les autres, mais si la collectivité comme telle a progressé. Ensuite la praxis de la libération repose sur la nécessité de la lutte et de l'affrontement avec l'adversaire. Dans une image de type libéral, les chances sont égales pour tous et l'échec est attribuable aux défaillances de l'individu. Dans une praxis de libération, la force occulte est celle du dehors et seule l'identification de l'adversaire et la lutte ouverte ont chance de dénouer la situation. Dans la théologie de la libération, les références bibliques sont légion, rappelant que le salut s'appelle aujourd'hui libération et que seule une lutte dans l'histoire peut rendre actuel l'Exode. Chaque peuple dominé est pour aujourd'hui le peuple hébreu, esclave en Égypte. Et si Dieu a fait une alliance avec ce peuple pour lui donner la dignité, la liberté, une terre et une prospérité, chaque peuple qui se libère reprend à son compte l'aventure biblique. La croyance au salut oblige au courage historique, au service de la libération, laquelle est comme une préfiguration de ce salut. Si le christianisme a prêché la soumission, c'est parce qu'il avait perdu une partie de sa force de protestation. Dans la mesure où la situation actuelle est le résultat d'une forme d'injustice, le christianisme, comme source de générosité et d'idéal moral, oblige à la lutte pour la libération. Pour les Québécois, la libération nationale est le premier objectif à poursuivre dans un vaste projet de ce genre.

\section{Le fond de la question}

$\underline{\text { Retour à la table des matières }}$

Comme on le voit, les arguments se renvoient presque l'un à l'autre, mais leurs grandes références symboliques sont assez variables. La thèse fédéraliste se réfère de manière privilégiée à une symbolique humaniste, gravitant autour des thèmes de la paix et de l'entente. La thèse séparatiste se réfère de façon privilégiée à des symboliques collectives plus axées sur le changement et la liberté.

De ce point de vue, il me semble que nous vivons des moments privilégiés puisque nous voici affrontés à de grandes questions morales dans une culture qui serait plutôt marquée par l'ennui.

En poursuivant plus avant notre réflexion, nous en arrivons à penser que l'hypothèse de l'indépendance québécoise relance l'interrogation éthique par la radicalité des questions qu'elle pose. 
D'abord elle pose la question du projet de société. Malgré les discours mythiques (à la Freud, à la Rousseau) sur l'origine de la société comme pacte originel, pour l'être humain qui naît la société est un déjà-là qui le précède et le pré-détermine. Notre milieu social n'est pas extérieur à nous. Il est une partie de nous-mêmes. Il nous influence, nous façonne, nous offre des clés culturelles d'interprétation de l'existence, nous situe dans des rapports économiques et des relations sociales, nous offre une langue, un art de vivre, une géographie. La société est le champ dans lequel l'individualité se construit. Bien sûr, le rapport de la personne à sa communauté d'appartenance est dialectique. Il est fait d'héritage et de rupture, d'acquiescement et de dissidence. L'individu peut être fier de sa communauté, en avoir honte, la défendre, la refuser. Normalement, il en va d'elle comme de sa propre existence, à savoir qu'on ne peut la changer sans l'avoir au préalable bien identifiée et ratifiée.

Un projet révolutionnaire qui ne serait que refus n'aurait au fond aucune chance de succès.

Sur ce préambule, quels seraient les éléments fondamentaux de la question?

D'abord, il me semble que le projet d'indépendance nationale se double d'un projet social. La force et la profondeur du P.Q., son ambiguïté également, reposent dans la jonction de ces deux dimensions. Il ne peut y avoir de projet national québécois qui n'implique en même temps une nouvelle négociation des rapports sociaux à l'intérieur du Québec. C'est la différence fondamentale entre le nationalisme québécois actuel et le nationalisme traditionnel qui défendait les Canadiens français au nom des idéaux du conservatisme. Le programme du P.Q. se veut une affirmation d'indépendance dans un projet de type social-démocratique. À l'opposé, la nouvelle gauche québécoise issue de la révolution tranquille ne manifeste qu'un intérêt mitigé au projet d'indépendance et insiste davantage sur la refonte radicale du projet social. On sait que la première théorie marxiste est fondamentalement internationaliste puisqu'elle fait appel à tous les travailleurs pour une modification radicale des rapports de production sans tenir compte des autres réalités historiques quelles qu'elles soient. Il y a eu, par exemple, des discussions très vives entre Proudhon et Karl Marx sur les stratégies à développer pour l'établissement du socialisme, Marx se faisant le défenseur de l'Internationale (dans un contexte plutôt allemand et anglais !) tandis que Proudhon demeurait plus préoccupé de projets de libération des travailleurs axés sur la réalité nationale. Ainsi, l'évolution de la Commune de Paris (1871) sera très différente selon qu'on la verra d'un point de vue français ou d'un point de vue internationaliste.

Le nationalisme québécois actuel, dans sa phrase politique militante, lie et dissocie à la fois les deux questions. Quand il les dissocie, il annonce soit un projet socialiste qui fait peur au monde, soit un projet nationaliste qui apparaît chez les gens de gauche comme la promesse d'un nouveau fascisme ou, plus simplement, la substitution d'une bourgeoisie francophone à une bourgeoisie 
anglophone ni meilleure ni pire que la précédente, et à tout prendre, plutôt pire que la précédente parce que plus naïve.

Toutefois, dans la mesure où le nationalisme est porteur d'une énergie, le contenu de sa dynamique ne peut pas se réduire à la seule promesse d'un pays pour les gens d'ici. Il véhicule celle d'un nouveau type de pays dans une nouvelle façon de vivre. Les prophètes de l'Ancien Testament ne faisaient pas autrement qui annonçaient une terre pour les gens en exil et un nouveau type d'exercice de la politique qui bannirait l'injustice, défendrait en priorité le pauvre, la veuve et l'orphelin, ferait cesser les pratiques frauduleuses des commerçants et réorienterait les objectifs militaires trop confiants dans la puissance des chevaux, en faveur d'un nouvel âge spirituel dans la paix.

Au Québec, l'énergie libérée par l'aspiration indépendantiste doit en partie servir de tremplin à changer les rapports sociaux. Rapports économiques d'abord, en améliorant les services publics, en rétrécissant les différences d'échelle de revenus (en France, ils sont encore de un à onze alors qu'en Hollande, ils seraient de un à quatre), en abolissant le chômage, en assurant la reconnaissance des droits de l'homme dans une définition renouvelée, en accordant des recours aux individus lésés, etc. On pourrait ajouter à ce nouveau projet social une dimension écologique pour que la nouvelle manière de vivre au Québec aille dans le sens d'une harmonie retrouvée avec la nature, une meilleure utilisation des ressources, une insistance sur la qualité de vie plutôt qu'une course effrénée pour une consommation maximale. La promesse d'un Québec nouveau ne peut à la fois apporter la liberté, la justice et une motoneige à chaque individu !

Il me semble, au plan théorique, que les deux dimensions nationale ou sociale sont bien liées. Les agents du conservatisme ont, en un sens, raison de lier le fédéralisme à la sauvegarde du statu quo. C'est exactement l'argument qui a prévalu en 1867 pour la création du Canada (contre l'opinion du Parti libéral du temps !). Toute libération a des tendances libertaires. Le retentissement éthique de la question nationale c'est donc la possibilité qu'elle fait surgir d'une nouvelle négociation du contexte social. En vérité le contexte social est toujours instable et discuté. Mais une dynamique de changement politique important fait surgir une brèche propice à des ajustements vraiment nouveaux des rapports de classe dans une société donnée.

La deuxième question que nous apporte la thèse nationaliste est celle de l'identité personnelle et collective. C'est un thème cher aux chansonniers que celui de l'absence de patrie. Le Québécois ne sait plus qui il est dans la multitude de ses appartenances. L'élite intellectuelle traditionnelle pouvait ou s'identifier au groupe culturel anglais (Wilfrid Laurier, Louis Saint-Laurent, Pierre Trudeau sont des exemples achevés de ce bilinguisme de promotion) ou même, plus subtilement, à la France (les gens qui parlent et vivent à la française); plus rares sont ceux qui savent d'où ils sont et où ils vont. Surtout dans le milieu montréalais, l'identité francophone semble une identité menacée. L'hégémonie économique du milieu anglophone, le malaise scolaire, la 
question des immigrants ont déterminé une crise radicale de l'identité. Le francophone montréalais se demande : «Que sera la survivance française quand Montréal sera devenu majoritairement anglais et qu'il ne restera plus que des îlots français, des enclaves françaises à Montréal et en province ?» Quand même le Saguenay ou la ville de Québec seraient francophones à 95\%, ils ne feront pas le poids si Montréal avec sa puissance culturelle a basculé dans le bassin anglophone.

Ceci nous ramène à une question radicale, la plus profonde de toutes. C'est quoi être humain ? Pendant plusieurs années, j'ai pensé vraiment que l'assimilation était notre seule voie raisonnable. Être Chinois, Japonais, Espagnol ou Algérien, cela a-t-il vraiment une importance ? À ce niveau d'abstraction, non. Ce n'est pas être plus homme qu'être blanc. Mais nul n'est homme sans être d'abord de tel ou tel pays, de telle ou telle culture. Il n'y a pas d'être humain abstrait. On est noir, ou blanc, ou jaune, ou mulâtre. On ne peut flotter hors l'humanité dans une essence abstraite. Un être humain n'est pas un destin théorique dans l'univers des principes. C'est une réalité terriblement incarnée, avec les limites que cette situation impose mais aussi dans la richesse que donne cette expérience. Sous cet aspect, chaque langue, chaque culture, chaque société dit et réalise l'humanité, l'accomplit en un lieu donné. Elle la limite aussi, mais cette limite même fait partie intégrante de la réalité et constitue une dimension de l'expérience. Quand certaines cultures ne voient ailleurs que des limites et chez elles une pure humanité sans restriction, nous sommes alors en plein impérialisme !

Les Québécois sont donc tenus de dire qui ils sont et qui ils veulent être. Être français c'est une manière de vivre, de penser, de sentir, d'accomplir le destin spirituel de l'existence humaine. Être anglais aussi. Nous pouvons décider de cesser d'être français pour devenir anglophones ou américains. Au niveau individuel, des pans entiers de la race canadienne-française l'ont fait. En général, ça coûte deux ou trois générations. Défi redoutable et aventureux, dont témoigne, par exemple, l'œuvre de Jack Kerouac. La nation francophone du Canada, ou du Québec, peut faire ce choix et décider, ou de s'affirmer politiquement dans un Québec indépendant, ou de poursuivre le statu quo (plus ou moins modifié) dans le Canada actuel, ou même de s'assimiler. Il y aurait de très bons arguments dans chacun de ces choix. Je pense, pour ma part, que l'originalité culturelle constitue une des richesses humaines et que l'expérience québécoise est l'exploration d'une des facettes possibles du destin de l'humanité. Ce n'est pas un drame de décider nous-mêmes qu'il en soit autrement. Mais j'ai tendance à penser qu'il est de notre responsabilité et de notre devoir de poursuivre notre expérience de francophone en terre d'Amérique et d'aller au bout de cette logique.

Dans quelle mesure un peuple est-il tenu de défendre son identité, de la poursuivre, de lutter pour elle ? À moins de se réfugier derrière un providentialisme naïf, on ne peut en appeler à une volonté divine : « le ciel a marqué sa carrière en ce monde nouveau » (O Canada, deuxième couplet. À signaler que la version anglaise n'est pas une traduction !). Mais cette contingence 
historique qui nous fait blanc ou noir, homme ou femme, vivant dans ce siècle plutôt que dans un autre, quel est son sens ? Une simple fatalité, un devoir, un hasard? Pour une part, elle doit pour chacun être une chance, c'est-à-dire la chance primordiale d'un accomplissement au sein de cette réalité. Tout changement qui ne serait que fuite ou négation de l'origine me paraîtrait une évasion et donc une démission. Je ne récuse pas a priori le changement. Mais je pense que le changement ne peut être accompli que dans une volonté d'assumer. La question fataliste du « pourquoi je suis ainsi ?» (fils de tel père, avec telle hérédité, etc.) ne peut recevoir de réponse que dans la décision : «je décide qu'étant ce que je suis, j'agirai maintenant de telle façon ».

De manière générale, l'appartenance historique collective va de soi. Elle est soutenue par une ou des idéologies et se construit sur plusieurs appartenances de niveau inférieur. On le voit bien encore aujourd'hui en France alors que ce pays demeure pour les Français une patrie qui n'a pas anéanti les régionalismes ni même les résistances à l'égard des forces dominantes qui ont construit la France actuelle contre des réalités régionales très fortes (même chose en Italie, en Allemagne ou aux États-Unis). Mais dans un pays qui marche bien, l'identité collective ne fait pas vraiment question. Elle repose sur une culture, une géographie, une langue, un état, souvent une religion. Elle est entretenue par des expériences historiques, des guerres, des conflits sociaux et politiques, des fêtes. Elle est enfin supportée par un système idéologique.

Il serait intéressant, si nous en avions le temps, d'analyser les supports idéologiques mis de l'avant dans le système canadien et le système québécois pour construire l'une ou l'autre appartenance. Mais le seul fait du conflit et de la possibilité même d'une question référendaire font bien voir que l'identité n'est pas acquise actuellement et que la décision, dans un sens ou dans l'autre, va en réalité contenir un projet d'identité. En rigueur de ternies, il n'y a pas sur ce point de principe d'éthique. Il y a un droit fondamental à l'autodétermination, droit acquis au plan international, droit humain que, par exemple, et l'Église catholique et le Conseil des Églises canadiennes ont reconnu. Si le Québec le veut, il peut faire, en droit, son autonomie et l'on ne peut réduire sa mutation à une sécession. Mais s'il le fait, ou ne le fait pas, dans quelle mesure prend-il une décision sur son identité future ? Et quel est alors le poids de cette décision sur sa continuité, sur sa fidélité aux ancêtres, sur sa propre conscience historique ? Ce n'est pas être plus humain qu'être québécois ou canadien. Mais accomplir son humanité en un sens ou en un autre, c'est tout de même une décision importante dont on ne peut dire le retentissement a priori.

Sans bien connaître l'histoire des peuples, si je me rappelle mes lettres grecques et romaines, le discours idéologique sur la survivance historique fait partie du discours essentiel sur la dignité humaine. Ni dans l'Eliade, ni dans l'Odyssée, ni chez Tite-Live, ni chez Cicéron, ni même chez saint Augustin, on ne laisse soupçonner que la contingence historique de l'appartenance à un peuple pourrait n'être qu'une question pragmatique. Elle est toujours enveloppée d'une aura magique. Elle est traversée par une symbolique tragique. On 
meurt pour sa patrie. En perdant son histoire et son identité, on perd comme une partie de son essence. L'apatride est un sous-homme. Sauvegarder son identité c'est comme un «devoir », une nécessité parfois absurde pourtant toujours hautement digne. C'est comme s'il y avait une noblesse à garder intacte l'origine, à ne pas laisser se perdre la particularité qui constitue une nation. En écologie, la disparition d'une espèce revêt le caractère d'un drame. Dans l'histoire humaine aussi. Chez nous, dans les milieux nationalistes chauvins, l'évidence de milieu, c'est que l'existence francophone est noble et digne ; l'existence anglophone apparaît comme une sous-culture, un antihumanisme. C'est là une prétention assez affligeante, digne des histoires de «newfies », assez proche du racisme sectaire... Mais elle révèle en creux la perception d'un apport irremplaçable de la culture spécifique d'un groupe dans la définition de l'être humain. Grâce à chaque culture, nous savons un peu mieux ce qu'est l'humanité. Par elle, le mystère humain se dévoile et révèle ses significations ultimes.

Dans l'idéologie de la fin du $19 \mathrm{e}$ siècle et du début du $20 \mathrm{e}$, le rôle providentiel des Canadiens français c'est de sauvegarder la tradition et de fournir au monde des missionnaires. L'échec historique est alors surmonté par un rôle internationaliste, les Canadiens français jouant un rôle messianique et divin dans l'humanité. Dans la crise de la sécularisation, c'est par des raisons humaines que doit se justifier l'identité. En quoi et comment pouvons-nous et devons-nous nous affirmer historiquement? Notre expérience a-t-elle suffisamment de corps, est-elle suffisamment signifiante pour justifier un nouveau projet historique. L'identité livrée par l'histoire a-t-elle un sens existentiel actuel suffisant pour servir de tremplin à un nouveau projet qui engage maintenant l'avenir ? Chose étonnante, et ce n'est pas le moindre de ses paradoxes, la crise québécoise et le projet indépendantiste surgissent au moment de la baisse foudroyante de la natalité et de la crise de la sécularisation. D'un côté, les Québécois ne veulent plus se reproduire mais semblent désirer s'identifier collectivement : la baisse de natalité peut avoir alors le sens \&une volonté suicidaire comme celui d'une déception à l'égard des impasses historiques de l'actuel statut - indépendamment de toutes les raisons raisonnables par ailleurs. De l'autre côté, la chute d'un certain univers religieux renvoie le destin historique à ses propres raisons de vivre.

Bref, les Québécois ne peuvent trouver dans des principes éternels ni dans une révélation la réponse à leur question, mais on ne peut nier que la question qu'ils se posent soit hautement morale ni qu'elle ait pour l'avenir des retentissements éthiques importants. 


\section{Des développements ultérieurs?}

$\underline{\text { Retour à la table des matières }}$

Dans certains milieux, on signale que la crise québécoise peut rendre un grand service à l'humanité, par exemple, dans l'élaboration d'un modèle québécois de socialisme ou dans la création d'une société véritablement francophone en Amérique. Je trouve à ces théories des relents de missionnarisme. C'est d'abord pour eux-mêmes que les Québécois doivent choisir, pour des raisons intrinsèques à leur propre situation. C'est là seulement que leur geste pourra avoir un sens international. On ne peut décemment penser que l'hypothèse de la séparation puisse avoir de grands retentissements sur l'éthique contemporaine, même en nous maintenant au seul niveau de la culture. Nous avons beau posséder maintenant une littérature, une musique, des chansonniers et quelques éléments d'une industrie culturelle, nous ne sommes tout de même pas, par nous-mêmes, une culture dominante. Mais nous sommes un cas d'espèce, Toynbee l'avait déjà reconnu. Nous apportons un quelque chose, si minime soit-il. Et il est raisonnable de penser, au moins pour nous, que le débat actuel va déterminer pour de longues années à venir le discours éthique. À la morale domestique du christianisme traditionnel, à la poussée personnaliste de l'existentialisme et de la psychologie moderne, la crise politique va substituer une thématique de caractère collectif qui tendra à devenir dominante. Dans quelle mesure cette interrogation prendra-t-elle corps dans des praxis repérables et pourra-t-elle guider des choix quotidiens ? Plusieurs de mes amis moralistes déplorent la «perte de la fibre morale » chez mes concitoyens, comme si la nouvelle perspective qui naît permettrait de critiquer et d'évacuer les anciennes exigences sans vraiment imposer de « sacrifices » et de « conversions » pour maintenant. On a beau être «quelque chose comme un grand peuple », un peuple ne survit pas si les individus qui le composent n'investissent pas une part considérable de leur propre vie dans l'existence commune. Si chacun fraude au dépend de l'État, ne pense qu'à son profit étroit et ne rêve qu'à des vacances en Floride, il n'y a pas grand espoir.

L'hypothèse de la séparation a tendance actuellement à être dramatisée et à faire surgir des militances partisanes et passionnées. Elle est lourde, de part et d'autre, d'une certaine violence, d'une intransigeance. Je suis, pour ma part, plutôt porté à y voir l'émergence d'une nouvelle générosité, Dans les années qui viennent, nos politiciens auront-ils le courage de faire appel davantage à cette militance courageuse faite de gratuité et de conviction, ou la rentrée des grands intérêts favorisera-t-elle plutôt l'engagement massif de mercenaires ? Nous aurions alors perdu une bonne chance de devenir meilleurs que notre 
propre cœur. À bien y penser, en parodiant Clémenceau, on pourrait dire que la politique est une chose beaucoup trop sérieuse pour la confier aux politiciens. La question politique est une question morale. Et c'est à la conscience des hommes et des femmes d'ici qu'elle s'adresse ultimement !

Fin du texte 